\title{
Acute dyspnea in Emergency Department: point of care ultrasound in the diagnosis of atrial sarcoma
}

\author{
Maria Immacolata Arnone, ${ }^{1}$ Giampiero Foccillo, ${ }^{2}$ Federica De Pisapia, ${ }^{1}$ Giuliano De Stefano, ${ }^{1}$ \\ Giovanni Albano, ${ }^{1}$ Maria Viviana Carlino, ${ }^{3}$ Alfonso Sforza, ${ }^{3}$ Enrico Giuseppe Ruggiero, ${ }^{3}$ Costantino Mancusi ${ }^{1}$ \\ ${ }^{1}$ Hypertension Research Center, Federico II University Hospital, Napoli; ${ }^{2}$ Emergency Department, San Paolo Hospital, Napoli; \\ ${ }^{3}$ Emergency Department CTO, Napoli, Italy
}

\begin{abstract}
We describe a rare case of a 65-year-old patient presented to Emergency Department for acute dyspnea. Traditional diagnostic work-up was done and the emergency physician promptly performed a bedside point-of-care ultrasound. A giant mass in the left atrium was detected with impairing of mitral valve function. The patient was then sent to cardiac surgery department with a final diagnosis of high-grade cardiac sarcoma.
\end{abstract}

\section{Case Report}

A 65-year-old man presented to Emergency Department (ED) with exertional dyspnea for several months aggravated in the last days. He had no history of cardiovascular disease but reported episodes of chest pain in the last month.

At presentation blood pressure was 140/90 $\mathrm{mmHg}$, heart rate (HR) 75 bpm regular, oxygen saturation was $98 \%\left(\mathrm{FiO}_{2} \quad 34 \%\right)$ and respiratory rate was 28 breaths/min.

Chest auscultation revealed mild diastolic murmur (II/VI) on the mitral area. Physiological vesicular murmur was present during lung auscultation, partially abolished at base bilaterally. There was no peripheral edema. The patient was afebrile.

The electrocardiogram showed sinus rhythm (HR

Correspondence: Costantino Mancusi, Hypertension Research Center, Federico II University Hospital, Napoli, Italy.

E-mail: costantino.mancusi@unina.it

Key words: Point of care ultrasound; emergency ultrasound; dyspnea; atrial sarcoma; integrated ultrasound.

Conflict of interest: the authors declare no potential conflict of interest.

Received for publication: 22 August 2018.

Accepted for publication: 8 October 2018.

This work is licensed under a Creative Commons Attribution NonCommercial 4.0 License (CC BY-NC 4.0).

(C) Copyright M.I. Arnone et al., 2019

Licensee PAGEPress, Italy

Italian Journal of Medicine 2019; 13:45-47

doi:10.4081/itjm.2019.1081
$72 \mathrm{bpm}$ ), without specific conduction abnormalities. Blood tests showed white cell count in the normal range, hemoglobin of $13.4 \mathrm{mg} / \mathrm{dL}$, international normalized ratio of 1.11 , glomerular filtration rate of $90 \mathrm{~mL} / \mathrm{min} / 1.73 \mathrm{~m}^{2}$, C reactive protein of $0.45 \mathrm{mg} / \mathrm{dL}$ (normal range: $0.0-0.5 \mathrm{mg} / \mathrm{dL}$ ).

Arterial blood gas test showed a pH of 7.44; $\mathrm{pCO}_{2}$ $30 \mathrm{mmHg}$; $\mathrm{pO}_{2} 71 \mathrm{mmHg}$; bicarbonate $\left(\mathrm{HCO}_{3}^{-}\right) 20.4$ $\mathrm{mmol} / \mathrm{L}$; lactate $2.4 \mathrm{mmol} / \mathrm{L} ; \mathrm{P} / \mathrm{F}$ ratio was 208 . Electrolytes were within normal range.

Chest radiography revealed partial left costophrenic angle blunting (Figure 1).

A point-of-care ultrasound (POCUS) with a portable ultrasound device was performed at the bedside.

The study revealed a big echogenic mass in the left atrium $(52 \times 32 \mathrm{~mm})$, partially attached to the anterior mitral leaflet. During diastole the mass prolapsed through the mitral valve, producing a functional mitral stenosis with transvalvular mean gradient of $8 \mathrm{mmHg}$ (Figures 2 and 3). Left and right systolic functions were preserved. There was a moderate tricuspid regurgitation with a pulmonary systolic artery pressure of $60 \mathrm{mmHg}$. The inferior vena cava was of normal size but fixed during respiratory activity.

Lung ultrasound showed bilateral pleural effusion.

The patient underwent contrast-enhanced computed tomography scan of thorax that revealed the presence of bilateral pleural effusion, a partially calcified mass in the left atrium with contrast enhancement. Aorta and pulmonary veins showed no abnormality.

The patient was transferred to the department of cardiovascular surgery and urgent surgery was performed. The mass was excised and histological examination revealed high-grade sarcoma with chondromatous differentiation. The patient was then referred to the oncologic center for further evaluation. 


\section{Discussion}

Dyspnea is the most common symptom of patients presenting to ED. Careful anamnesis, physical examination, laboratory test and chest radiography represent the main tools for differential diagnosis. However, these techniques have a low accuracy and are often inconclusive. ${ }^{1}$

POCUS performed at the bedside represents a feasible and non-invasive procedure that can help clinicians to make a rapid diagnosis. The integrated evaluation of heart, lung and inferior vena cava allows differential diagnosis of dyspnea and represents an extension of clinical examination, even in rare cases such as intra cardiac mass that requires more costly imaging techniques. ${ }^{1,2}$

The differential diagnosis of intra cardiac mass includes thrombi, tumors and vegetation. Thrombi and vegetation are the most likely etiology.

Cardiac tumors are rare with secondary tumors that are 20 to 40 times more common than primary cardiac ones. The incidence of primary cardiac tumors is extremely low, ranging from $0.0017 \%$ to $0.019 \%$. Of these, about three-quarters are benign, commonly myxomas, and the remaining quarter is formed by malignant tumors that are predominantly sarcomas. ${ }^{3}$

Cardiac sarcomas can arise in any area of the heart, although angio-sarcoma is typically found in the right atrium, whereas fibro sarcoma and undifferentiated sarcoma in the left atrium.

In undifferentiated sarcomas a chondromatous differentiation can be detected and it is generally diagnostic of malignancy. ${ }^{4}$

Signs and symptoms of intra cardiac mass are nonspecific and highly variable in relation to localization, size and composition of the cardiac mass. Generally, clinical presentation includes pulmonary or systemic

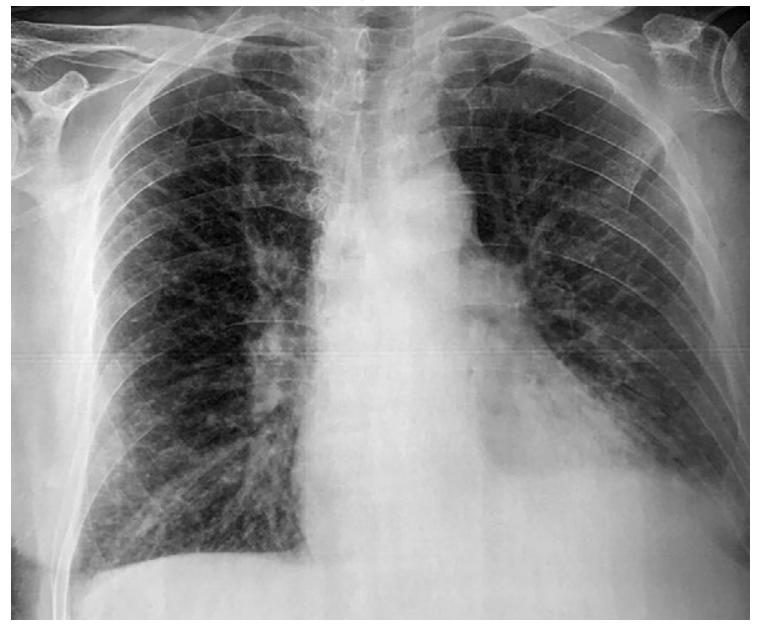

Figure 1. Chest X-ray.

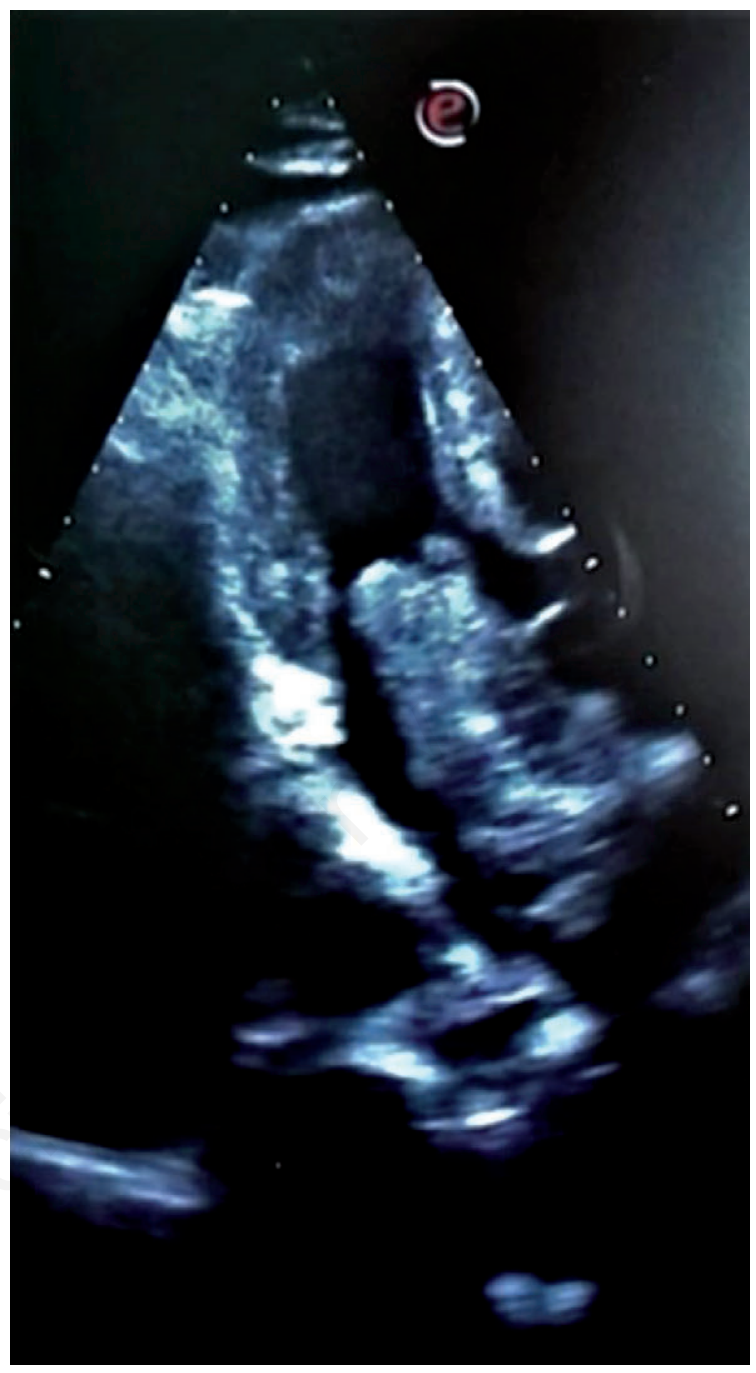

Figure 2. Apical three-chamber view of atrial sarcoma.

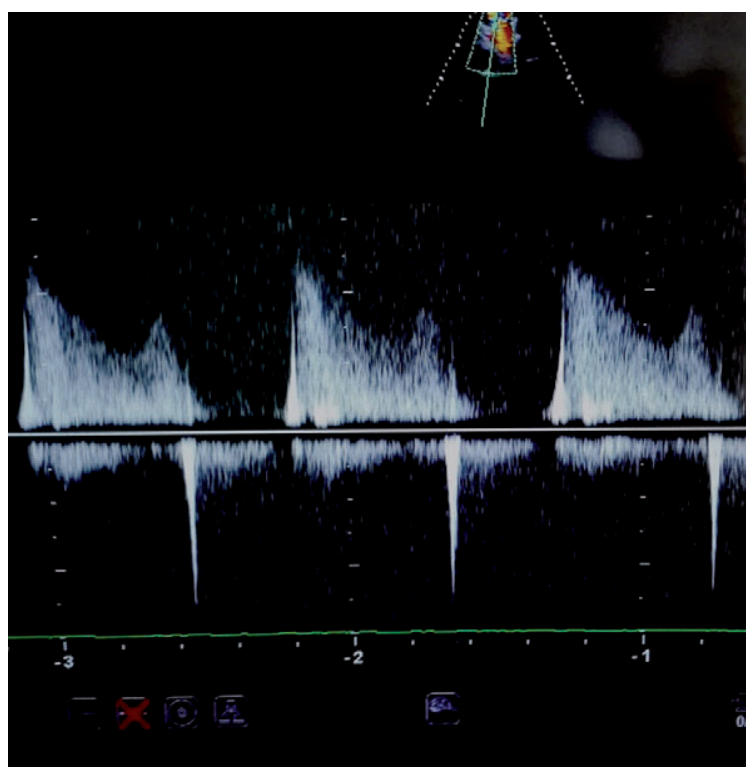

Figure 3. Continuous wave Doppler mitral valve. 
embolization, constitutional symptoms, arrhythmias and congestive heart failure from intra-cardiac obstruction. 5,6

We describe a dyspneic patient with a mass in the left atrium that impaired the mitral function causing a functional mitral valve stenosis. The patient developed also pulmonary artery hypertension.

POCUS performed at the bedside rapidly identified the pathophysiological condition of the patient and guided the second level diagnostic assessment.

\section{Conclusions}

This case emphasizes the important role of POCUS in the differential diagnosis of acute dyspnea. Rapid ultrasonographic assessment performed in Emergency department can help physicians to make a rapid diagnosis and guide an appropriate treatment, even in rare cases such as intra cardiac mass that requires more invasive and costly imaging techniques.

\section{References}

1. Zanobetti M, Scorpiniti M, Gigli C, et al. Point-of-care ultrasonography for evaluation of acute dyspnea in the emergency department. Chest 2017 [Epub ahead of print].

2. Sforza A, Mancusi C, Carlino MV, et al. Diagnostic performance of multi-organ ultrasound with pocketsized device in the management of acute dyspnea. Cardiovasc Ultrasound 2017;15:16.

3. Leja MJ, Shah DJ, Reardon MJ. Primary cardiac tumors. Tex Heart Inst J 2011;38:261-2.

4. Orlandi A, Ferlosio A, Roselli M, et al. Cardiac sarcomas: an update. J Thorac Oncol 2010;5:1483-9.

5. Vander Salm TJ. Unusual primary tumors of the heart. Semin Thorac Cardiovasc Surg 2000;12:89-100.

6. Hsieh CH, Seak CJ, Chiu TF, et al. An uncommon cause of heart failure: cardiac sarcomas in the left atrium. J Emerg Med 2011;40:e123-4. 